\title{
Alcoholic Hepatitis: A Review
}

\author{
Nooshin Hosseini ${ }^{1}$, Julia Shor ${ }^{1}$, and Gyongyi Szabo ${ }^{2}$ \\ ${ }^{1}$ University of Massachusetts, Gastroenterology, University of Massachusetts Medical School, and ${ }^{2}$ Professor, \\ Department of Medicine, University of Massachusetts, 364 Plantation Street, LRB-208, Worcester, MA, USA
}

Received 28 December 2018; Revised 5 April 2019; Editorial Decision 5 April 2019; Accepted 9 April 2019

\begin{abstract}
Alcoholic liver disease (ALD) represents a spectrum of injury, ranging from simple steatosis to alcoholic hepatitis to cirrhosis. Regular alcohol use results in fatty changes in the liver which can develop into inflammation, fibrosis and ultimately cirrhosis with continued, excessive drinking. Alcoholic hepatitis $(\mathrm{AH})$ is an acute hepatic inflammation associated with significant morbidity and mortality that can occur in patients with steatosis or underlying cirrhosis. The pathogenesis of ALD is multifactorial and in addition to genetic factors, alcohol-induced hepatocyte damage, reactive oxygen species, gut-derived microbial components result in steatosis and inflammatory cell (macrophage and neutrophil leukocyte) recruitment and activation in the liver. Continued alcohol and pro-inflammatory cytokines induce stellate cell activation and result in progressive fibrosis. Other than cessation of alcohol use, medical therapy of $\mathrm{AH}$ is limited to prednisolone in a subset of patients. Given the high mortality of $A H$ and the progressive nature of ALD, there is a major need for new therapeutic intervention for this underserved patient population.
\end{abstract}

\section{INTRODUCTION}

Alcoholic liver disease (ALD) is the leading cause of alcohol related deaths worldwide. Alcohol contributes to progression of other etiologies of liver disease, and increases the risk of developing hepatocellular carcinoma (Safdar and Schiff, 2004). As an integral part of the fabric of American culture, alcohol consumption is widespread and cuts across socio-economic, regional, and racial lines. Approximately $7 \%$ of adult Americans meet DSM-IV criteria for the diagnosis of alcohol abuse or alcohol dependence (Grant et al., 1992; Bowman, 2014; Samhsa, 2014; Agriculture, 2015).

Excessive alcohol consumption can cause a range of injury to the liver, from negligible to fatty infiltration, to cirrhosis over the course of several years (Lefkowitch, 2005). Sustained, excessive alcohol use can cause inflammatory changes in the liver, leading to more serious damage known as alcoholic steatohepatitis, or alcoholic hepatitis. A subset of these patients will eventually develop severe alcoholic hepatitis, which carries a much more dire short-term prognosis. Depending on the degree of inflammation and damage, these conditions may lead to fibrosis and eventually cirrhosis and liver failure (Teli et al., 1995).

The majority of heavy drinkers do not ultimately develop advanced liver disease. This variability in outcomes suggests that various factors, both inheritable and environmental, can contribute to or determine individual patients' susceptibility to advanced liver disease.

\section{PREVALENCE}

Alcoholic hepatitis (AH) represents a significant public health burden. In 2007, approximately 56,000 patients were hospitalized with $\mathrm{AH}$ across the United States, with an average associated cost of $\$ 37,769$ per hospitalization (Liangpunsakul, 2011). The mean age of those hospitalized was 53 years; nearly $75 \%$ of these patients were male.

The mortality rate associated with $\mathrm{AH}$ is staggering. Forty-four percent of all deaths from liver disease in 2003 were attributable to alcohol-induced disease (Yoon and Yi, 2006). One study conducted in Denmark from 1999-2008 found the 28 day mortality rate for patients with alcoholic hepatitis to be $14-24 \%$, with 5 year mortality rate of 56\% (Chedid, 1991; Sandahl et al., 2011).

\section{EPIDEMIOLOGY}

Several factors have been linked to development of ALD and AH. Alcoholic hepatitis is generally thought to occur with excessive drinking that takes place over a period of at least 20 years, manifesting in the 4th to 5th decades of life (Bellentani et al., 1997, Mezey et al., 1988). Recent studies have shown an increased risk of AH and cirrhosis among individuals drinking more than 30-60 g/day (Becker et al., 1996; Rehm et al., 2010). Patients admitted to the 
hospital with cirrhosis and alcoholic hepatitis report a history of drinking greater than $120 \mathrm{~g}$ on average per day (Lelbach, 1975; Mezey et al., 1988).

While there is clear evidence that prolonged, heavy alcohol use is associated with ALD, only $35 \%$ of excessive drinkers eventually develop steatohepatitis and $\mathrm{AH}$, and only $10 \%$ ultimately develop cirrhosis (Friedman, 2008). This suggests that other factors contribute to the overall risk (Liangpunsakul, 2011) (Table 1). These include the pattern of drinking, and type of alcohol consumed and genetic factors. Drinking outside of mealtimes, drinking spirits or beer, and binge drinking are associated with a higher risk of advanced ALD (Bellentani et al., 1997; Wechsler and Austin, 1998; Barrio et al., 2004; NIAAA NIoAAaA, 2004).

Gender also plays a notable role in ALD (Baraona et al., 2001). Women's risk for developing severe ALD increases with daily intake of $20-40 \mathrm{~g}$ of alcohol, while the threshold for men appears to be 60-80 g (Mezey et al., 1988; Rotily et al., 1990; Bellentani et al., 1997; Lu et al., 2004). Various mechanisms have been proposed in the literature to explain this phenomenon, rooted in differences in alcohol metabolism due to variability in ADH activity, body fat distribution, liver volume, and influence of sex hormones on inflammatory response (Frezza et al., 1990; Seitz et al., 1993; Ikejima et al., 1998; Colantoni et al., 2000).

There are significant geographic differences in the incidence of ALD. Rates of ALD are higher in eastern Europe, southern Europe, and the United Kingdom (Klatskin, 1961; Popova et al., 2007). The lowest rates of ALD are observed in countries with large Muslim populations. The consumption of alcohol in the United States falls in between (Rehm et al., 2013). Ethnicity may be considered a risk. In the US, Hispanics and African Americans show an increased risk of developing ALD compared to whites, and higher mortality from ALD (Stinson et al., 2001; Stewart, 2002).

Alcoholic hepatitis has a strong association with malnutrition (Mendenhall et al., 1995). Protein-calorie malnutrition has been shown to correlate with severity and outcomes of $\mathrm{AH}$, while high fat diets may have a protective effect (Mitchell and Herlong, 1986; Mishra et al., 1989; Mendenhall et al., 1995). Nutrient deficiencies have been implicated in the pathogenesis of severe ALD, including riboflavin, B12, vitamin A and zinc (Corrao et al., 1998). Zinc has been identified as decreasing gut mucosal permeability by disrupting tight junctions, which may play a role in the pathogenesis of $\mathrm{AH}$ (Zhong et al., 2010).

Obesity has also been identified as an independent risk factor for ALD, and is thought to potentiate severity of fatty liver disease in heavy alcohol intake (> 50 g/day) (Ekstedt et al., 2009; Hart et al., 2010; Liu et al., 2010; Takahashi et al., 2015; Sookoian and Pirola, 2016).

Other concomitant liver diseases may accelerate alcoholic liver disease, most significantly hepatitis C. Heavy alcohol consumption is believed to act synergistically with hepatitis $\mathrm{C}$ in the progression

Table 1. Disease modifiers for alcoholic liver disease

\begin{tabular}{ll}
\hline Disease modifiers & \\
\hline Alcohol intake & Genetics \\
Age & Obesity \\
Sex & Medications \\
Race & Drugs of abuse \\
Diet & Other liver diseases \\
Nutrition & Environmental exposure \\
\hline
\end{tabular}

of advanced liver disease (Corrao and Arico, 1998; Hutchinson et al., 2005). Furthermore, in alcoholic hepatitis, the presence of hepatitis $\mathrm{C}$ is an independent risk factor for higher mortality at 6 months (Punzalan et al., 2015).

Another factor thought to play a role in ALD risk is hepatic iron and has been identified as a predictor of mortality in alcoholic cirrhosis. The significance and mechanism of iron overload in the development of ALD and AH has not been definitively shown, and remains an area of interest (LeSage et al., 1983; Ganne-Carrie et al., 2000; Gleeson et al., 2006).

\section{GENETICS}

Genetic factors affect the risk of ALD as evidenced by monozygotic twin studies (Hrubec and Omenn, 1981; Stickel and Hampe, 2012). Studies on polymorphisms for gene coding for alcohol metabolism, including alcohol dehydrogenase, acetaldehyde dehydrogenase and cytochrome P450 2E1, genes regulating the innate immune response (i.e. IL-1, TNF), and the PNPLA3 gene, showed that certain variants are associated with the risk of alcoholism, and possible association with alcoholic cirrhosis (Khoruts et al., 1991; Menon et al., 2001).

\section{PATHOGENESIS OF ALCOHOLIC LIVER DISEASE}

The pathogenesis of alcoholic liver disease involves multiple factors including hepatocyte damage due to alcohol and its metabolites, cholestasis, recruitment and activation of innate immune cells by gutderived pro-inflammatory danger signals, Kupffer cells, and recruited macrophages and neutrophils in the liver. The perpetual presence of these factors during chronic excessive alcohol use triggers ineffective anti-inflammatory pathways and results in activation of stellate cells and myofibroblasts in the liver leading to fibrosis and alcoholic cirrhosis (Arteel and Crabb, 2016; Louvet and Mathurin, 2015) (Fig. 1).

During alcohol consumption, alcohol is quickly absorbed in the GI tract resulting in increased blood alcohol concentrations. While most of the alcohol is metabolized in the liver in hepatocytes, alcohol also has substantial direct effects on the GI tract. Alcohol is metabolized in hepatocytes by alcohol dehydrogenase into acetaldehyde, a highly toxic compound with a short half-life, then further by acetaldehyde dehydrogenase into acetate. Both of these key enzymes have a low $\mathrm{km}$ and become saturated above the level of about 3-4 drinks of alcohol and higher alcohol concentrations trigger alternate mechanisms for alcohol metabolism that include the Cytochrome

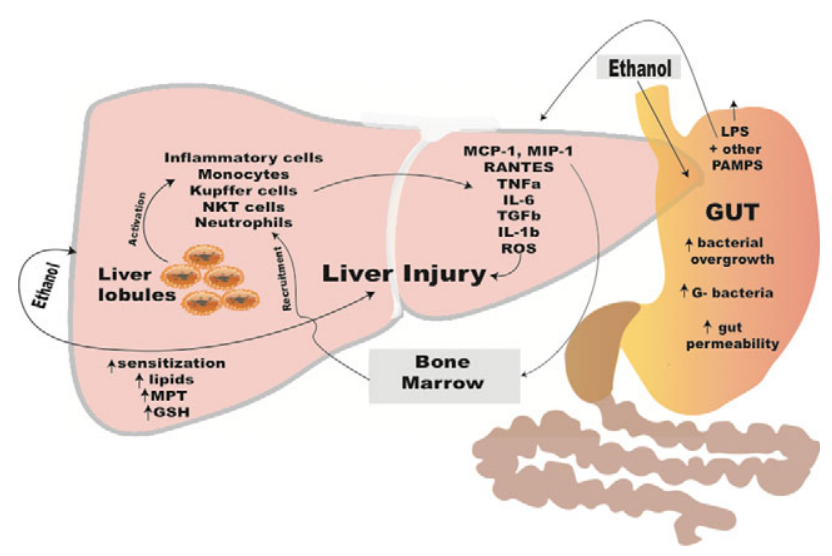

Fig. 1. Pathomechanisms of alcohol-induced liver injury involving the gutliver access. 
P4502E1 system. Upon repeated high doses of alcohol, CYP2E1 is upregulated resulting in accelerated metabolism. This process produces high levels of reactive oxygen species (ROS) in hepatocytes leading to reduced levels of intracellular antioxidants such as sadenosine methionine (SAME) and glutathione (GSH) (Gao and Bataller, 2011).

Acute binge drinking and chronic excessive alcohol consumption result in liver steatosis that is characterized by accumulation of fat in hepatocytes. Mechanistic studies revealed that key transcription factors such as hypoxia-inducible factor 1 (HIF-1) and regulators of fatty acid metabolism are affected by alcohol in hepatocytes that lead to steatosis (Nath et al., 2011). These include inhibition of $\operatorname{PPAR} \alpha$, and upregulation of ADRP (adpocyte differentiation-related protein, also known as perilipin-2), a protein that stabilizes lipid droplets in hepatocytes (Nath et al., 2011). Recent studies also revealed that alcohol results in damage and activation of apoptotic pathways in hepatocytes. Alcohol-induced endoplasmic reticulum (ER) stress activates (Stimulator of Interferon Genes) leading to phosphorylation of IRF3 (Interferon Regulatory Factor 3) that interacts with and activates the mitochondrial apoptotic pathways (Petrasek et al., 2013). Damaged hepatocytes release damageassociated molecular patterns (DAMPs) that are recognized by immune cells in the liver as 'danger' signals leading to a proinflammatory response. Some of these danger signals include uric acid and ATP; inhibition of these pathways in animal models of alcoholic liver disease ameliorated liver damage, steatosis and inflammation (Iracheta-Vellve et al., 2015).

A unique and major factor in the pathomechanism of ALD is related to its effects on the GI tract. Numerous studies have demonstrated that alcohol results in a 'leaky gut' via disrupting the tight junctions of the epithelial barrier permitting translocation of microbial components from the intestine to the portal circulation and the liver (Szabo and Bala, 2010). Alcohol use also affects the composition of the gut microbiome which is a contributing factor to ALD. Studies demonstrated that gut 'decontamination' with antibiotics significantly attenuates alcohol-related liver inflammation, steatosis and liver damage (Louvet and Mathurin, 2015). Lipopolysaccharide, a component of the outer wall of gram negative bacteria, is increased in the portal and systemic circulation after acute binge and chronic alcohol use in mice and humans (Szabo and Bala, 2010). LPS is recognized by the Toll-like receptor 4 complex expressed on immune cells and parenchymal cells in the liver and results in upregulation of the inflammatory cascade (Petrasek et al., 2010). In animal models of alcoholic liver disease, gut decontamination with antibiotics or elimination of liver Kupffer cells and macrophages attenuated alcoholic liver disease (Louvet and Mathurin, 2015).

While fat accumulation in hepatocytes characterizes early alcoholic liver disease, activation of the liver resident macrophages, Kupffer cells, is associated with chronic alcoholic liver disease. In addition, there is recruitment of circulating and bone marrowderived inflammatory cells (monocytes, macrophages and neutrophil leukocytes) into the liver that produce pro-inflammatory cytokines (TNFo, IL-1ß, IL-6, MCP1) (Petrasek et al., 2010; Szabo et al., 2012). The presence of neutrophil leukocytes is a histological hallmark of acute alcoholic hepatitis, correlating with clinical outcomes (Szabo et al., 2012). In acute alcoholic hepatitis, highly elevated circulating levels of pro-inflammatory cytokines, TNF $\alpha$ and IL-1ß, correlate with disease severity and survival (Szabo et al., 2012). Increased peripheral blood neutrophilia represents a diagnostic challenge in alcoholic hepatitis because it can be the result of disease-related inflammation in the absence of microbial infection.

Presence of inflammatory cells in the liver sinusoids results in activation of stellate cells characterized by loss of retinoic acid and induction of fibrogenic genes such as alpha-1 smooth muscle actin and collagen-1 production (Louvet and Mathurin, 2015). Activated stellate cells proliferate and produce collagen that contributes to liver fibrosis. Persistent and prolonged activation of hepatic stellate cells and myofibroblasts leads to progressive deposition of collagen, liver fibrosis and in advanced form, bridging fibrosis and cirrhosis.

Research in human, animal models and in vitro systems discovered many important aspects of the pathogenesis of alcoholic hepatitis, yet some critical questions remain unanswered. Why some and not all heavy drinkers develop ALD? What is the trigger for the most severe clinical form of alcoholic liver disease, acute alcoholic hepatitis? Why does alcoholic hepatitis progress even after cessation of alcohol use in most patients?

\section{DIAGNOSIS}

Diagnosis of alcoholic hepatitis poses a challenge as it is largely a diagnosis of exclusion. It is made via a combination of clinical suspicion, clinical presentation, laboratory, imaging, and biopsy data. Patients with alcoholic hepatitis frequently present with a combination of typical symptoms including jaundice, abdominal pain, fullness or distention, fever, GI bleeding, or changes in mental status. A recent consensus report defined clinical criteria for the diagnosis of alcoholic hepatitis (Crabb et al., 2016).

\section{PHYSICAL EXAM}

Exam findings in alcoholic hepatitis frequently include hepatomegaly, jaundice and fever. Presence of stigmata of chronic liver disease suggests underlying cirrhosis (Lischner et al., 1971; Mendenhall, 1981). Another possible finding in AH is abdominal bruit, which is thought to be caused by increased blood flow in the hepatic artery, however there is conflicting sensitivity and specificity reported in the literature (Sherman and Hardison, 1979; Han et al., 2002).

\section{LABORATORY FINDINGS}

Initial laboratory workup for suspected alcoholic liver disease includes CBC, INR, LFTs, GGT, carbohydrate deficient transferrin, and serologies for viral hepatitis. AST and ALT are typically less than $300 \mathrm{iu} / \mathrm{L}$ and rarely over 500 . The AST is typically higher than the ALT, and $70 \%$ of patients have an AST-ALT ratio of greater than 1.5 (Bell et al., 1994; Sorbi et al., 1999; Nyblom et al., 2004). Lab abnormalities in AH may also include an elevated bilirubin, typically greater than $5 \mathrm{mg} / \mathrm{dL}$, with a median of 13 , elevated INR and GGT, and low albumin and prealbumin (Poynard et al., 1984; Louvet et al., 2008). Other common hematologic findings include anemia, macrocytosis, leucopenia, lymphocytopenia, and thrombocytopenia (Bell et al., 1994; Mundle et al., 2000; Mathurin et al., 2002; Nguyen-Khac et al., 2011). Recently described biomarkers for chronic alcohol use can aid the diagnosis of AH (Anton and Moak, 1994; Berlakovich et al., 2004; Hock et al., 2005; Hietala et al., 2006; Niemela, 2007; Madhubala et al., 2013). 


\section{IMAGING}

Abdominal imaging studies, including ultrasound, CT and MRI, may be a useful tool for diagnosing ALD. Imaging findings in patients with AH include hepatomegaly, fatty changes in the liver, evidence of underlying cirrhosis, or ascites (Piekarski et al., 1980; Saverymuttu et al., 1986; Borra et al., 2009). Doppler flow studies of the hepatic artery can be useful as well, and may reveal an elevated peak systolic velocity, or an increase in vessel diameter (Han et al., 2002).

\section{BIOPSY}

Diagnosis based on clinical criteria alone carries a $10-50 \%$ risk of misclassification of alcoholic hepatitis (Kryger et al., 1983; Mookerjee et al., 2011). EASL Practical Guidelines on Alcoholic Liver Disease recommends the use of liver biopsy in patients with suspected severe alcoholic hepatitis (Mookerjee et al., 2011). Biopsy is recommended in patients with a clinical picture of $\mathrm{AH}$ and any of the following factors: 1 . Hypotension/massive bleeding on admission, 2. Sepsis at admission, 3. Suspicion of malignant liver disease based on clinical and/or imaging criteria, 4. Uncertain assessment of alcohol drinking history, 5. Cocaine use in the preceding 3 months, 6. Recent use of a potential hepatotoxic substance (Mundle et al., 2000). Consensus of US experts recommends liver biopsies in all patients where the clinical diagnosis of ALD or AH is uncertain.

\section{HISTOLOGY}

Histologic findings can vary based on stage of the disease. Typical features include steatosis, confluent parenchymal necrosis, intrasinusoidal and pericentral collagen, ballooning degeneration, lobular inflammation affecting perivenular regions (in the earliest stages), Mallory bodies (amorphous eosinophilic inclusion bodies) surrounded by neutrophils, foamy degeneration of hepatocytes and cholestasis (Edmondson et al., 1967; MacSween and Burt, 1986; Lefkowitch, 2005) (Suppl. Table 1).

Findings of alcoholic liver disease, including perivenular and pericellular fibrosis, which often coexist with $\mathrm{AH}$, may portend future cirrhosis, especially in patients who are co-infected with hepatitis C or continue to drink (Worner and Lieber, 1985; Hall, 1994).

Megamitochondria may be present in milder forms of $\mathrm{AH}$ and is associated with a lower incidence of cirrhosis, fewer complications and good long-term survival prospects (Chedid et al., 1986). Increased severity of inflammation (i.e. degree of PMN infiltration and cholestatic changes) is associated with a poor prognosis. Degree of inflammation can also predict response to steroid treatment (Marbet et al., 1987; Nissenbaum et al., 1990; Mathurin et al., 1996) (Suppl. Table 2).

\section{COMPLICATIONS}

The short-term mortality associated with AH is significant - 30$50 \%$ at 3 months. Patients with severe AH can develop encephalopathy, and may require airway protection. One of the most serious complications is acute kidney injury, often can be attributed to hepatorenal syndrome, which is associated with a very poor prognosis. Early volume expansion with albumin or crystalloid should be a priority in patients with creatinine elevated above baseline (Altamirano et al., 2012).

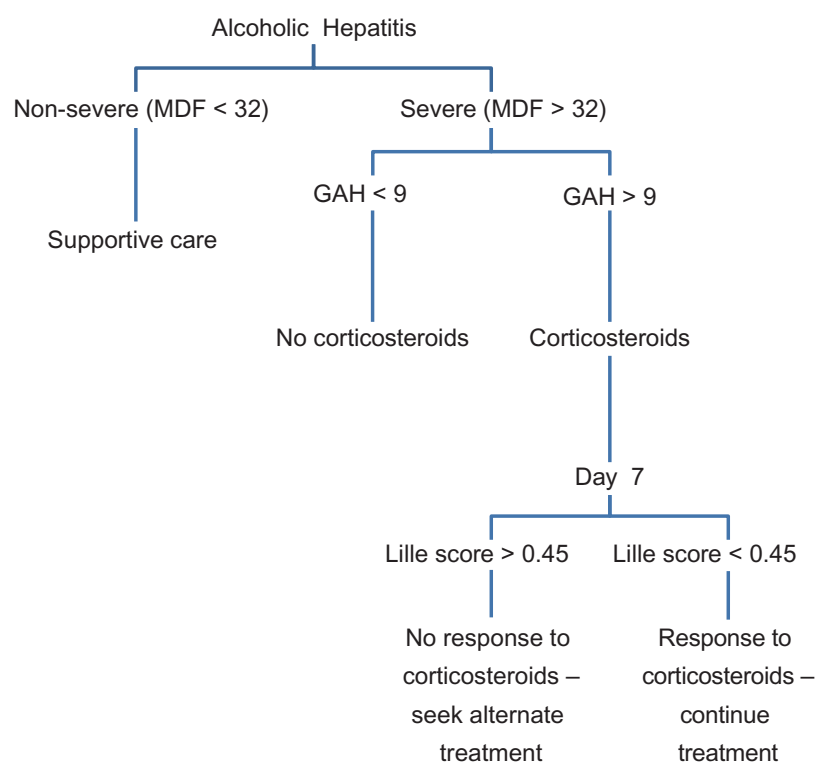

Fig. 2. Treatment algorithm for corticosteroids in alcoholic hepatitis using Maddrey, GAH, and Lille scores.

Infections are also very common. All patients should be screened for infection, particularly when considering therapy with corticosteroids. SBP should be ruled out in all patients with ascites.

A significant number of patients with severe alcoholic hepatitis fail to recover despite treatment and abstinence from alcohol. If recovery has not been reached by 3 months despite the above measures, then the chances of spontaneous recovery are low, and may lead to death. AH can also progress to cirrhosis.

While not necessarily a direct consequence of $\mathrm{AH}$, given that patients with AH likely have underlying ALD, it is important to recognize the increased potential for developing hepatocellular carcinoma (HCC). ALD significantly increases the risk of HCC. The risk for HCC is 3.6 times higher in those with any level of alcohol consumption, as compared to those who abstain from alcohol consumption entirely (Ascha et al., 2010). Among patients with alcoholic cirrhosis, 1-2\% develop HCC (Seitz and Stickel, 2007). Furthermore, excess alcohol use may act synergistically with hepatitis $\mathrm{C}$ infection, hepatitis $\mathrm{B}$, or obesity to accelerate progression towards HCC (Zakhari, 2013).

\section{MODELS FOR DISEASE SEVERITY, TREATMENT GUIDANCE AND PREDICTION OF SURVIVAL}

Decisions regarding treatment of alcoholic hepatitis are contingent upon the patient's prognosis. A number of scoring models for alcoholic hepatitis (discussed below) have been developed to assess severity of disease, predict mortality, and guide treatment (Fig. 2).

The oldest are the Child-Turcotte-Pugh (CTP) and MELD (now sodium-MELD). A Sodium-MELD score of 21 implies a 90 day mortality probability of $20 \%$, and has been used as a threshold for starting corticosteroids in AH (Kamath et al., 2001; Farnsworth et al., 2004). While both scores are used for assessing gravity of disease and mortality, they are less beneficial in guiding prognosis and treatment in AH (Suppl. Table 2) (Fig. 3).

Two scores have been approved to define severity of the disease and to determine when coritocosteroid therapy is recommended - 


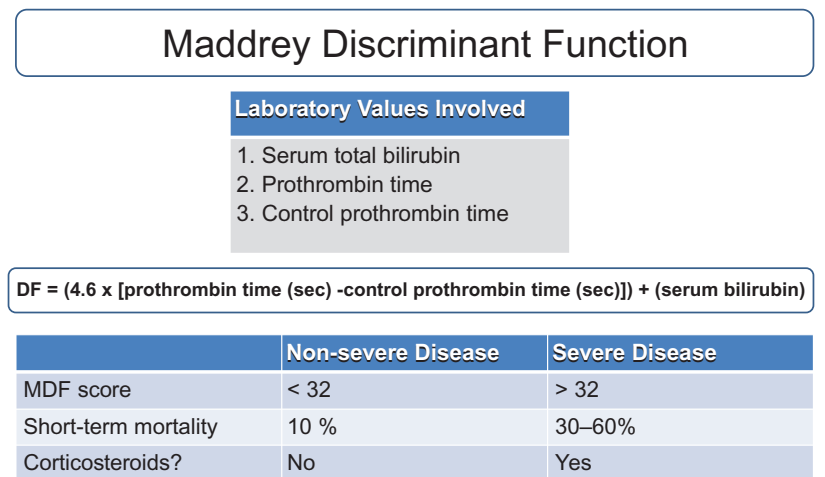

Fig. 3. Maddrey Discriminant Function (MDF). Scores are used to predict mortality and for guiding treatment with coritcosteroids.

the Maddrey Discriminant Function score (MDF) and the Glasgow Alcoholic Hepatitis Score (GAHS).

The Maddrey Discriminant Function score is the most widely used scoring system for AH. It functions as a guide for treatment, predictor of severity of disease, and as a predictor of individual mortality. The calculation incorporates both serum bilirubin and PT at time of diagnosis. Disease is classified as severe $(\mathrm{DF}>32)$ and nonsevere $(\mathrm{DF}<32)$. Short-term mortality for a $\mathrm{DF}<32$ is predicted at $10 \%$, and $30-60 \%$ for DF $>32$. As such, patients who fall within the 'severe' category are most likely to benefit from treatment (see Treatment section below) (Maddrey et al., 1978). This scoring system is the most widely used in determining whether or not to start corticosteroid treatment (Fig. 4).

The Glasgow Alcoholic Hepatitis Score was developed for use as a more accurate predictor of overall outcomes than the MDF. Given the potential contraindications to corticosteroid therapy, GAHS helps select patients with severe alcoholic hepatitis who would most benefit from treatment. The score is a function of age, leukocyte count, serum urea, bilirubin, and PT. A score above 9 is associated with a poor prognosis. Patients with both MDF $>32$ and a GAHS $>9$ treated with corticosteroids showed improvements in both 28 day and 84 day survival. Conversely, patients with a MDF $<32$ and a GAHS of $<9$ treated with corticosteroids had no survival benefit (Forrest et al., 2005) (Suppl. Table 4).

The ABIC score predicts the 3-month mortality of patients with $\mathrm{AH}$, but is not widely used to predict severity of disease or for starting corticosteroids. It is calculated using age, bilirubin, INR and creatinine. The ABIC score further stratifies the severity of alcoholic hepatitis into low $(<6.71)$, intermediate (6.71-8.99), and high (>9.0) classes. These classes correspond to a 90 -day mortality of $0 \%, 30 \%$, and $75 \%$, respectively (Dominguez et al., 2008) (Suppl. Table 3).

In addition to assessing severity of patients with $\mathrm{AH}$, the Lille score is used to assess response of patients to steroid treatment. The calculation uses age, albumin, PT, and bilirubin on day 0 of treatment. It also takes into account the presence or absence of renal insufficiency and the change in bilirubin at day 7 of treatment. A score of $<0.45$ is associated with a $15 \%$ mortality at 6 months, while a score of 0.45 or greater predicts a $75 \%$ mortality. After corticosteroids are initiated, a score of 0.45 or greater on day 7 indicates a lack of response to treatment (Louvet et al., 2007). There is also data to suggest that the Lille score at day 4 can be also predictive of response to treatment. Initial scoring systems were dependent upon factors on the day of presentation. However, as evidenced by

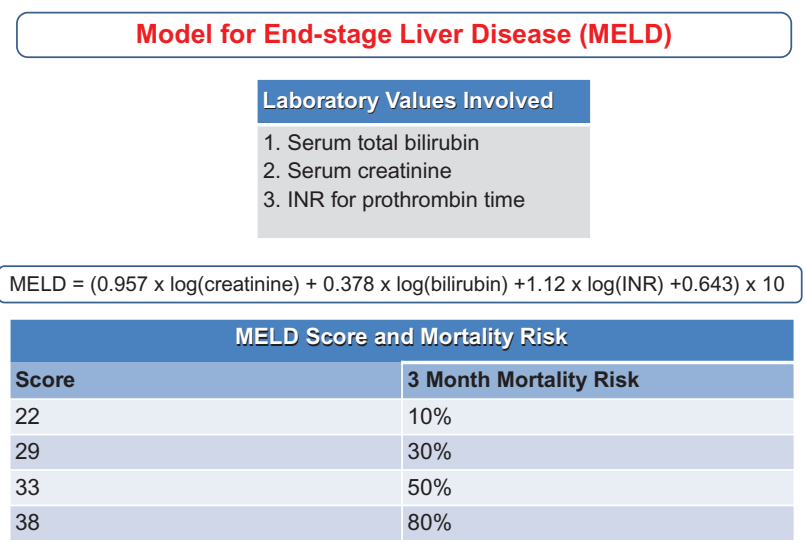

Fig. 4. Model for End-stage liver disease (MELD) scoring system.

the Lille score, early improvement in liver function impacts shortterm mortality, and thus repeated testing and calculation of scores may be useful during hospitalization (Suppl. Table 5).

Recently, new scoring systems that incorporate histologic information have been developed. The Alcoholic Hepatitis Histologic Score (AHHS) incorporates 4 pathologic findings from biopsy specimens that are independently associated with survival. These include the stage of fibrosis, neutrophil infiltration, evidence of cholestasis, and presence of megamitochrondria. Patients are then stratified into categories of mortality within 90 days (Altamirano et al., 2014).

Per EASL 2018 guidelines, of the above scoring models, only two are approved to define the severity of $\mathrm{AH}$ and thus to establish when steroids should be initiated - the MDF and GAHS scores. Along the same lines, strict rules for cessation of therapy should be applied, by identifying early non-response to corticosteroid treatment, by using the Lille score (European Association for the Study of the Liver, 2018).

\section{TREATMENT}

The standard of care for patients with alcoholic liver disease and alcoholic hepatitis has not changed in the last several decades, yet mortality of patients with alcoholic hepatitis remains high. This demonstrates a major gap in development of new therapies and highlights the lack of attention to the alcoholic hepatitis patient population.

\section{TREATMENT OF ALCOHOLIC LIVER DISEASE AND MODERATE ALCOHOLIC HEPATITIS}

Patients with high risk and/or excessive alcohol use should be consulted to stop alcohol use. Alcohol cessation is difficult or unachievable for most patients due to addiction (Lazo and Mitchell, 2016). In the absence of full alcohol cessation, reduction of alcohol use not to exceed 2 drinks in men and 1 drink in women per day could be an acceptable initial intervention. Treating alcohol addiction is most effective with psychiatric help or participation in formal withdrawal programs. Medical interventions for alcohol addiction should also be considered.

There is no specific treatment for alcoholic liver disease at this time. Patients should be advised that continued alcohol use confers high risk of developing alcoholic hepatitis. In patients with decades or longer of excessive alcohol use, the likelihood of alcoholic liver 
fibrosis and cirrhosis increases and cirrhosis may develop even after cessation of alcohol use. Women should be advised about the greater probability of developing ALD and cirrhosis at shorter duration and doses of alcohol use compared to men even after stopping alcohol use (Lazo and Mitchell, 2016).

Previous studies showed no or limited benefit of antioxidants or PTU. Given the massive activation of pro-inflammatory cytokines in severe alcoholic hepatitis, anti-TNF therapies were used in experimental settings however showed no early benefit. All of the studies involving TNF $\alpha$ or TNF receptor blockade had to be discontinued due to the increased rate of infections (Petrasek and Szabo, 2016). This is not unexpected as chronic excessive alcohol users are immunocompromised and have poor anti-bacterial and anti-viral host defense mechanisms. Small studies found that administration of SAME as antioxidant had some benefits. In patients with severe alcoholic hepatitis, administration of $\mathrm{N}$-acetyl-cystamine (NAC) also provided benefit. Administration of a probiotic, lactobacillus GG, or zinc were shown to improve gut permeability in animal models and patients with alcoholic liver disease.

Moderate alcoholic hepatitis is clinically defined as elevated AST, AST/ALT ratio greater than 1.5, increased bilirubin and MELD $<19$ in the setting of recent excessive alcohol use and no other etiologies of liver disease (Crabb et al., 2016). As of 2018, there is no specific treatment for moderate alcoholic hepatitis. Some providers use pentoxifylline for 28 days but there are studies in patients with moderate and severe alcoholic hepatitis that show limited benefit to support this practice. The most effective therapy associated with improvement of alcoholic hepatitis is alcohol cessation.

\section{TREATMENT OF SEVERE ALCOHOLIC HEPATITIS}

Severe alcoholic hepatitis is defined as Maddrey discriminant factor $>32$ and/or MELD > 20 (Crabb et al., 2016). These patients are typically hospitalized and often require intensive care due to their risk for sepsis-like clinical presentation, cardiovascular instability, infection, all complicated by alcohol withdrawal in most acute presentations. The clinical syndrome of acute alcoholic hepatitis is characterized by a sepsis-like presentation due to sterile inflammation and cytokine 'storm' in the absence of a clear source of infection. The diagnostic challenge is that the low grade fever, tachycardia, and hypotension may also be heralding infection in these patients. Development of multi-organ involvement, most frequently hepatorenal syndrome and ARDS requiring mechanical ventilation, is associated with poor clinical outcome. Standard of care in the US in patients with acute alcoholic hepatitis (MDF $>32$ ) is prednisolone $40 \mathrm{~g}$ for 28 days, shown to improve 6 month mortality (Petrasek and Szabo, 2016). Several studies have evaluated pentoxifylline alone in severe alcoholic hepatitis however results were controversial (Petrasek and Szabo, 2016). Co-administration of prednisolone and pentoxifylline failed to provide added benefit over steroids alone, with the exception of reduction in hepatorenal syndrome (Thursz et al., 2015).

Patients with acute severe alcoholic hepatitis are managed by active supportive therapy. Intake of at least 3,000 daily calories is associated with improved survival (Petrasek and Szabo, 2016). If steroids were started, evaluation of the Lille score on day 7 has good prognostic value (Louvet et al., 2007).

In addition to the limited benefit of steroid therapy, it carries increased risk of infections and its use is limited to a narrow patient population. In patients with hepatic encephalopathy, recent acute GI bleeding and established or suspected infection, steroids should be avoided. There is a major need for new and effective therapy in alcoholic hepatitis, and several recent clinical trials are ongoing in the United States and other countries to develop new therapies. Some of the new interventions aim to inhibit inflammation by administration of a recombinant IL-1 receptor antagonist, IL-22 to improve gut barrier function and liver immunity, or use an orally administered FXR agonist that affects bile acid metabolism, gut permeability and presumably inflammation (Szabo, 2017).

Finally, the rapid deterioration of patients with chronic alcoholic hepatitis prompted some liver transplant centers to consider liver transplantation as a last resource (Mathurin et al., 2011). A recent study from Europe demonstrated a high success rate in patients who received liver transplantation (Mathurin et al., 2011). Both graft and recipient survival were average or better than in liver transplantation for other indications and alcohol recurrence was avoided in most patients with close postoperative management (Mathurin et al., 2011).

\section{TREATMENT OF ALCOHOL ADDICTION}

The most challenging aspect regarding care of patients with alcoholic hepatitis is alcohol addiction that results in craving and often, relapse. The care of patients before and after hospital discharge should include plans for mitigating alcohol addiction that may include different strategies such as behavioral treatments and/or voluntary admission to an alcohol withdrawal program, participation in Alcohol Anonymous (AA) and/or pharmaceutical drugs to reduce physical symptoms of addiction and alcohol craving. The drugs Acomprosate and Naltrexone are approved for the treatment of Alcohol Use Disorder (AUD) and are most usually used although have limited effects (Miller et al., 2011). The safety and efficacy of most of these drugs have been established in addicted individuals who had no concurrent diagnosis of alcoholic liver disease or alcoholic hepatitis. The use of Acomprosate is safe in patients with existing liver disease however, Naltrexone has potential liver toxicity in patients with existing liver disease. There are other emerging drugs not approved in all countries like Topiramate, Ondasentron, Gabapentin, Varenicline and Baclofen. These drugs are NOT approved for AUD in the USA (although baclofen is now approved in France only for AUD), but their use could be considered as an off-label treatment in a case-by-case scenario. Furthermore, baclofen is the only medication formally tested in patients with advanced liver disease, as shown by a few clinical trials (Addolorato et al., 2007; Morley et al., 2018). Of special interest for this review, a retrospective study conducted at UCLA indicated the safety and utility of baclofen in AUD patients with alcoholic hepatitis when the total bilirubin level approaches $10 \mathrm{mg} / \mathrm{dl}$ (Yamini et al., 2014).

There are several cognitive-behavioral (CBT) therapy options for substance abuse disorders, including alcohol addiction that should be considered in patients with alcoholic liver disease and alcoholic hepatitis (McHugh et al.; and NIAAA website). These can be used alone or combined with pharmacologic treatments. Brief interventions that involve 1-4 short counseling sessions with a trained interventionist can help to reduce alcohol use (NIAAA website). Motivational interviewing (MI) is an approached that is based on assessing ambivalence toward behavior change in alcohol use. MI is usually offered in an individual format. Motivational enhancement therapy (MET) helps individuals to resolve their ambivalence about engaging the treatment for their alcohol use disorder (NIAAA website). MET can be successful in alcohol addicted people to improve their engagement in treatment and reduces their problem drinking (Principles of Drug Addiction Treatment, 2019a, Principles of Drug Addiction Treatment, 2019b; Alcohol Alert, 2005; McHugh et al., 2010). 


\section{CONCLUDING REMARKS}

Research in human, animal models and in vitro systems discovered many important aspects of the pathogenesis of alcoholic hepatitis, yet some critical questions remain unanswered. The standard of care for patients with alcoholic liver disease and alcoholic hepatitis has not changed in the last several decades, yet mortality of patients with alcoholic hepatitis remains high (15-50\%). This demonstrates a major gap in development of new therapies and highlights the lack of attention to alcoholic hepatitis. The primary means for therapy for patients with alcoholic hepatitis involves corticosteroids, and is limited to a narrow patient population, and has limited benefit and a high side effect profile. More recent therapies aim to inhibit inflammation by targeting cytokine mediated pathways, and by improving gut barrier function. Finally, the rapid deterioration of patients with alcoholic hepatitis has prompted some centers to consider transplantation as a last resort, which remains controversial. It is clear that given the grim outcome and excessive costs associated with alcoholic hepatitis despite major advances in medical therapies over the past several decades, however, there is a wide gap between our understanding of alcoholic hepatitis and treatment, and a glaring need for new therapeutic advancements in this area.

\section{SUPPLEMENTARY MATERIAL}

Supplementary data are available at Alcohol And Alcoholism online

\section{CONFLICT OF INTEREST STATEMENT}

Dr. Szabo consults for and received grants from Allergan. She consults for Terra Firma, Glympse Bio, Quest, Arrow, GLG, Salix, and Tobira. She received grants from Gilead, Genfit, Intercept, Verlyx, Novartis, SignaBlok, and Shire. She holds intellectual property rights with Up to Date. The co-authors declare no conflict of interest.

\section{FINANCIAL SUPPORT}

This work was supported by National Institutes of Health Grant U01 AA021893 (to G.S.)

\section{REFERENCES}

(NIAAA) NIoAAaA. (2014) NIAAA Council Approves Definition of Binge Drinking. NIAAA Newsletter, No. 3, Winter 2004.

(SAMHSA) SAaMHSA. (2014) National Survey on Drug Use and Health (NSDUH). Table 2.41B-Alcohol use in lifetime, past year, and past month among persons aged 18 or older, by demographic characteristics: Percentages, 2013 and 2014.

Addolorato G, Leggio L, Ferrulli A, et al. (2007) Effectiveness and safety of baclofen for maintenance of alcohol abstinence in alcohol-dependent patients with liver cirrhosis: randomised, double-blind controlled study. Lancet 370:1915-22.

Agriculture USDoHaHSaUSDo. (2015) Dietary Guidelines for Americans 2015-2020.

Alcohol Alert. Brief Interventions. National Institute of Health. National Institute on Alcohol Abuse and Alcoholism. 2005 (66). https://pubs.niaaa. nih.gov/publications/aa66/aa66.htm

Altamirano J, Fagundes C, Dominguez M, et al. (2012) Acute kidney injury is an early predictor of mortality for patients with alcoholic hepatitis. Clin Gastroenterol Hepatol 10:65-71.e3.
Altamirano J, Miquel R, Katoonizadeh A, et al. (2014) A histologic scoring system for prognosis of patients with alcoholic hepatitis. Gastroenterology 146:1231-9.e1-6.

Anton RF, Moak DH. (1994) Carbohydrate-deficient transferrin and gammaglutamyltransferase as markers of heavy alcohol consumption: gender differences. Alcohol Clin Exp Res 18:747-54.

Arteel GE, Crabb DW. (2016) Pathogenesis of alcoholic liver disease. In Chalasani N, Szabo G (eds). Alcoholic and Non-Alcoholic Fatty Liver Disease: Bench to Bedside. Switzerland: Springer International Publishing, 41-70.

Ascha MS, Hanouneh IA, Lopez R, et al. (2010) The incidence and risk factors of hepatocellular carcinoma in patients with nonalcoholic steatohepatitis. Hepatology 51:1972-8.

Baraona E, Abittan CS, Dohmen K, et al. (2001) Gender differences in pharmacokinetics of alcohol. Alcohol Clin Exp Res 25:502-7.

Barrio E, Tome S, Rodriguez I, et al. (2004) Liver disease in heavy drinkers with and without alcohol withdrawal syndrome. Alcohol Clin Exp Res 28:131-6.

Becker U, Deis A, Sorensen TI, et al. (1996) Prediction of risk of liver disease by alcohol intake, sex, and age: a prospective population study. Hepatology 23:1025-9.

Bell H, Tallaksen CM, Try K, et al. (1994) Carbohydrate-deficient transferrin and other markers of high alcohol consumption: a study of 502 patients admitted consecutively to a medical department. Alcohol Clin Exp Res 18:1103-8.

Bellentani S, Saccoccio G, Costa G, et al. (1997) Drinking habits as cofactors of risk for alcohol induced liver damage. The Dionysos Study Group Gut 41:845-50.

Berlakovich GA, Soliman T, Freundorfer E, et al. (2004) Pretransplant screening of sobriety with carbohydrate-deficient transferrin in patients suffering from alcoholic cirrhosis. Transpl Int 17:617-21.

Borra RJ, Salo S, Dean K, et al. (2009) Nonalcoholic fatty liver disease: rapid evaluation of liver fat content with in-phase and out-of-phase MR imaging. Radiology 250:130-6.

Bowman SA, Clemens JC, Friday JE, et al. (2014) Food Patterns Equivalents Database 2011-12: Methodology and User Guide. Food Surveys Research Group, Beltsville Human Nutrition Research Center, Agricultural Research Service. Beltsville, Maryland: US Department of Agriculture.

Chedid A, Mendenhall CL, Gartside P, et al. (1991) Prognostic factors in alcoholic liver disease. VA Cooperative Study Group. Am J Gastroenterol 86:210-6.

Chedid A, Mendenhall CL, Tosch T, et al. (1986) Significance of megamitochondria in alcoholic liver disease. Gastroenterology 90:1858-64.

Colantoni A, La Paglia N, De Maria N, et al. (2000) Influence of sex hormonal status on alcohol-induced oxidative injury in male and female rat liver. Alcohol Clin Exp Res 24:1467-73.

Corrao G, Arico S. (1998) Independent and combined action of hepatitis C virus infection and alcohol consumption on the risk of symptomatic liver cirrhosis. Hepatology 27:914-9.

Corrao G, Torchio P, Zambon A, et al. (1998) Alcohol consumption and micronutrient intake as risk factors for liver cirrhosis: a case-control study. The Provincial Group for the study of Chronic Liver Disease. Ann Epidemiol 8:154-9.

Crabb DW, Bataller R, Chalasani NP, et al. (2016) Standard Definitions and Common Data Elements for Clinical Trials in Patients With Alcoholic Hepatitis: Recommendation From the NIAAA Alcoholic Hepatitis Consortia. Gastroenterology 150:785-90.

Dominguez M, Rincon D, Abraldes JG, et al. (2008) A new scoring system for prognostic stratification of patients with alcoholic hepatitis. Am J Gastroenterol 103:2747-56.

Edmondson HA, Peters RL, Frankel HH, et al. (1967) The early stage of liver injury in the alcoholic. Medicine (Baltimore) 46:119-29.

Ekstedt M, Franzen LE, Holmqvist M, et al. (2009) Alcohol consumption is associated with progression of hepatic fibrosis in non-alcoholic fatty liver disease. Scand J Gastroenterol 44:366-74. 
European Association for the Study of the Liver. (2018) Electronic address EEE, European Association for the Study of the LEASL Clinical Practice Guidelines: Management of alcohol-related liver disease. J Hepatol 69: 154-81.

Farnsworth N, Fagan SP, Berger DH, et al. (2004) Child-Turcotte-Pugh versus MELD score as a predictor of outcome after elective and emergent surgery in cirrhotic patients. Am J Surg 188:580-3.

Forrest EH, Evans CD, Stewart S, et al. (2005) Analysis of factors predictive of mortality in alcoholic hepatitis and derivation and validation of the Glasgow alcoholic hepatitis score. Gut 54:1174-9.

Frezza M, di Padova C, Pozzato G, et al. (1990) High blood alcohol levels in women. The role of decreased gastric alcohol dehydrogenase activity and first-pass metabolism. N Engl J Med 322:95-9.

Friedman SL. (2008) Mechanisms of hepatic fibrogenesis. Gastroenterology 134:1655-69.

Ganne-Carrie N, Christidis C, Chastang C, et al. (2000) Liver iron is predictive of death in alcoholic cirrhosis: a multivariate study of 229 consecutive patients with alcoholic and/or hepatitis $\mathrm{C}$ virus cirrhosis: a prospective follow up study. Gut 46:277-82.

Gao B, Bataller R. (2011) Alcoholic liver disease: pathogenesis and new therapeutic targets. Gastroenterology 141:1572-85.

Gleeson D, Evans S, Bradley M, et al. (2006) HFE genotypes in decompensated alcoholic liver disease: phenotypic expression and comparison with heavy drinking and with normal controls. Am J Gastroenterol 101: 304-10.

Grant BF, Harford TC, Dawson DA, et al. (1992) Prevalence of DSM-IV alcohol abuse and dependence: United States, 1992. Alcohol Health Res World 18:243-8.

Hall PD. (1994) Pathological spectrum of alcoholic liver disease. Alcohol Alcohol Suppl 2:303-13.

Han SH, Rice S, Cohen SM, et al. (2002) Duplex Doppler ultrasound of the hepatic artery in patients with acute alcoholic hepatitis. J Clin Gastroenterol 34:573-7.

Hart CL, Morrison DS, Batty GD, et al. (2010) Effect of body mass index and alcohol consumption on liver disease: analysis of data from two prospective cohort studies. BMJ 340:c1240.

Hietala J, Koivisto H, Anttila P, et al. (2006) Comparison of the combined marker GGT-CDT and the conventional laboratory markers of alcohol abuse in heavy drinkers, moderate drinkers and abstainers. Alcohol Alcohol 41:528-33.

Hock B, Schwarz M, Domke I, et al. (2005) Validity of carbohydratedeficient transferrin (\%CDT), gamma-glutamyltransferase (gamma-GT) and mean corpuscular erythrocyte volume $(\mathrm{MCV})$ as biomarkers for chronic alcohol abuse: a study in patients with alcohol dependence and liver disorders of non-alcoholic and alcoholic origin. Addiction 100: 1477-86.

Hrubec Z, Omenn GS. (1981) Evidence of genetic predisposition to alcoholic cirrhosis and psychosis: twin concordances for alcoholism and its biological end points by zygosity among male veterans. Alcohol Clin Exp Res 5:207-15. Spring.

Hutchinson SJ, Bird SM, Goldberg DJ. (2005) Influence of alcohol on the progression of hepatitis $\mathrm{C}$ virus infection: a meta-analysis. Clin Gastroenterol Hepatol 3:1150-9.

Ikejima K, Enomoto N, limuro Y, et al. (1998) Estrogen increases sensitivity of hepatic Kupffer cells to endotoxin. Am J Physiol 274:G669-76.

Iracheta-Vellve A, Petrasek J, Satishchandran A, et al. (2015) Inhibition of sterile danger signals, uric acid and ATP, prevents inflammasome activation and protects from alcoholic steatohepatitis in mice. J Hepatol 63: 1147-55.

Kamath PS, Wiesner RH, Malinchoc M, et al. (2001) A model to predict survival in patients with end-stage liver disease. Hepatology 33:464-70.

Khoruts A, Stahnke L, McClain CJ, et al. (1991) Circulating tumor necrosis factor, interleukin-1 and interleukin- 6 concentrations in chronic alcoholic patients. Hepatology 13:267-76.

Klatskin G. (1961) Alcohol and its relation to liver damage. Gastroenterology 41:443-51.
Kryger P, Schlichting P, Dietrichson O, et al. (1983) The accuracy of the clinical diagnosis in acute hepatitis and alcoholic liver disease. Clinical versus morphological diagnosis. Scand J Gastroenterol 18:691-6.

Lazo M, Mitchell MC. (2016) Epidemiology and risk factors for alcoholic liver disease: bench to bedside. In Chalasani N, Szabo G (eds). Alcoholic and Non-Alcoholic Liver Disease. Switzerland: Springer International Publishing, 1-20.

Lefkowitch JH. (2005) Morphology of alcoholic liver disease. Clin Liver Dis 9:37-53.

Lelbach WK. (1975) Cirrhosis in the alcoholic and its relation to the volume of alcohol abuse. Ann N Y Acad Sci 252:85-105.

LeSage GD, Baldus WP, Fairbanks VF, et al. (1983) Hemochromatosis: genetic or alcohol-induced? Gastroenterology 84:1471-7.

Liangpunsakul S. (2011) Clinical characteristics and mortality of hospitalized alcoholic hepatitis patients in the United States. J Clin Gastroenterol, 45, 714-9.

Lischner MW, Alexander JF, Galambos JT. (1971) Natural history of alcoholic hepatitis. I. The acute disease. Am J Dig Dis 16:481-94.

Liu B, Balkwill A, Reeves G, et al. (2010) Body mass index and risk of liver cirrhosis in middle aged UK women: prospective study. BMJ 340:c912.

Louvet A, Diaz E, Dharancy S, et al. (2008) Early switch to pentoxifylline in patients with severe alcoholic hepatitis is inefficient in non-responders to corticosteroids. J Hepatol 48:465-70.

Louvet A, Mathurin P. (2015) Alcoholic liver disease: mechanisms of injury and targeted treatment. Nat Rev Gastroenterol Hepatol 12:231-42.

Louvet A, Naveau S, Abdelnour M, et al. (2007) The Lille model: a new tool for therapeutic strategy in patients with severe alcoholic hepatitis treated with steroids. Hepatology 45:1348-54.

Lu XL, Luo JY, Tao M, et al. (2004) Risk factors for alcoholic liver disease in China. World J Gastroenterol 10:2423-6.

MacSween RN, Burt AD. (1986) Histologic spectrum of alcoholic liver disease. Semin Liver Dis 6:221-32.

Maddrey WC, Boitnott JK, Bedine MS, et al. (1978) White RI, Jr. Corticosteroid therapy of alcoholic hepatitis. Gastroenterology 75: 193-9.

Madhubala V, Subhashree AR, Shanthi B. (2013) Serum carbohydrate deficient transferrin as a sensitive marker in diagnosing alcohol abuse: a case - control study. Journal of clinical and diagnostic research: JCDR 7: 197-200.

Marbet UA, Bianchi L, Meury U, et al. (1987) Long-term histological evaluation of the natural history and prognostic factors of alcoholic liver disease. J Hepatol 4:364-72.

Mathurin P, Duchatelle V, Ramond MJ, et al. (1996) Survival and prognostic factors in patients with severe alcoholic hepatitis treated with prednisolone. Gastroenterology 110:1847-53.

Mathurin P, Mendenhall CL, Carithers RL Jr., et al. (2002) Corticosteroids improve short-term survival in patients with severe alcoholic hepatitis (AH): individual data analysis of the last three randomized placebo controlled double blind trials of corticosteroids in severe AH. J Hepatol 36: 480-7.

Mathurin P, Moreno C, Samuel D, et al. (2011) Early liver transplantation for severe alcoholic hepatitis. N Engl J Med 365:1790-800.

McHugh RK, Hearon BA, Otto MW. (2010) Cognitive behavioral therapy for substance use disorders. Sychiatr Clin North Am 33:511-25.

Mendenhall CL. (1981) Alcoholic hepatitis. Clin Gastroenterol 10:417-41.

Mendenhall CL, Moritz TE, Roselle GA, et al. (1995) Protein energy malnutrition in severe alcoholic hepatitis: diagnosis and response to treatment. The VA Cooperative Study Group \#275. JPEN J Parenter Enteral Nutr 19:258-65.

Menon KV, Gores GJ, Shah VH. (2001) Pathogenesis, diagnosis, and treatment of alcoholic liver disease. Mayo Clin Proc 76:1021-9.

Mezey E, Kolman CJ, Diehl AM, et al. (1988) Alcohol and dietary intake in the development of chronic pancreatitis and liver disease in alcoholism. Am J Clin Nutr 48:148-51.

Miller PM, Book SW, Stewart SH. (2011) Medical treatment of alcohol dependence: a systematic review. Int J Psychiatry Med 42:227-66. 
Mishra L, Sharma S, Potter JJ, et al. (1989) More rapid elimination of alcohol in women as compared to their male siblings. Alcohol Clin Exp Res 13: $752-4$.

Mitchell MC, Herlong HF. (1986) Alcohol and nutrition: caloric value, bioenergetics, and relationship to liver damage. Annu Rev Nutr 6:457-74.

Mookerjee RP, Lackner C, Stauber R, et al. (2011) The role of liver biopsy in the diagnosis and prognosis of patients with acute deterioration of alcoholic cirrhosis. J Hepatol 55:1103-11.

Morley KC, Baillie A, Fraser I, et al. (2018) Baclofen in the treatment of alcohol dependence with or without liver disease: multisite, randomised, double-blind, placebo-controlled trial. Br J Psychiatry 212:362-9.

Mundle G, Munkes J, Ackermann K, et al. (2000) Sex differences of carbohydrate-deficient transferrin, gamma-glutamyltransferase, and mean corpuscular volume in alcohol-dependent patients. Alcohol Clin Exp Res 24:1400-5.

Nath B, Levin I, Csak T, et al. (2011) Hepatocyte-specific hypoxia-inducible factor-1alpha is a determinant of lipid accumulation and liver injury in alcohol-induced steatosis in mice. Hepatology 53:1526-37.

Nguyen-Khac E, Thevenot T, Piquet MA, et al. (2011) Glucocorticoids plus $\mathrm{N}$-acetylcysteine in severe alcoholic hepatitis. N Engl J Med 365:1781-9.

Niemela O. (2007) Biomarkers in alcoholism. Clin Chim Acta 377:39-49.

Nissenbaum M, Chedid A, Mendenhall C, et al. (1990) Prognostic significance of cholestatic alcoholic hepatitis. VA Cooperative Study Group \#119. Dig Dis Sci 35:891-6.

Nyblom H, Berggren U, Balldin J, et al. (2004) High AST/ALT ratio may indicate advanced alcoholic liver disease rather than heavy drinking. Alcohol Alcohol 39:336-9.

Petrasek J, Iracheta-Vellve A, Csak T, et al. (2013) STING-IRF3 pathway links endoplasmic reticulum stress with hepatocyte apoptosis in early alcoholic liver disease. Proc Natl Acad Sci U S A 110:16544-9.

Petrasek J, Mandrekar P, Szabo G Toll-like receptors in the pathogenesis of alcoholic liver disease. Gastroenterol Res Pract. 2010;2010.

Petrasek J, Szabo G. (2016) Treatment of Alcoholic Liver Disease Including Emerging Therapies, Novel Targets, and Liver Transplantation. In Chalasani N, Szabo G (eds). Alcoholic and Non-Alcoholic Fatty Liver Disease. Switzerland: Springer International Publishing, 291-312.

Piekarski J, Goldberg HI, Royal SA, et al. (1980) Difference between liver and spleen CT numbers in the normal adult: its usefulness in predicting the presence of diffuse liver disease. Radiology 137:727-9.

Popova S, Rehm J, Patra J, et al. (2007) Comparing alcohol consumption in central and eastern Europe to other European countries. Alcohol Alcohol 42:465-73.

Poynard T, Zourabichvili O, Hilpert G, et al. (1984) Prognostic value of total serum bilirubin/gamma-glutamyl transpeptidase ratio in cirrhotic patients. Hepatology 4:324-7.

Principles of Drug Addiction Treatment: A Research-Based Guide (Third Edition) Cognitive-Behavioral Therapy (Alcohol, Marijuana, Cocaine, Methamphetamine, Nicotine). National Institute of Health. National Institute on Drug Abuse. (January 2018) Advancing Addiction Science 2019a. https://www.drugabuse.gov/publications/principles-drug-addictiontreatment-research-based-guide-third-edition/evidence-based-approaches-todrug-addiction-treatment/behavioral

Principles of Drug Addiction Treatment: A Research-Based Guide (Third Edition) Motivational Enhancement Therapy (Alcohol, Marijuana, Nicotine). National Institute of Health. National Institute on Drug Abuse. (January 2018) Advancing Addiction Science 2019b. https://www. drugabuse.gov/publications/principles-drug-addiction-treatment-researchbased-guide-third-edition/evidence-based-approaches-to-drug-addictiontreatment/behavioral-2

Punzalan CS, Bukong TN, Szabo G. (2015) Alcoholic hepatitis and HCV interactions in the modulation of liver disease. J Viral Hepat 22:769-76.
Rehm J, Samokhvalov AV, Shield KD. (2013) Global burden of alcoholic liver diseases. J Hepatol 59:160-8.

Rehm J, Taylor B, Mohapatra S, et al. (2010) Alcohol as a risk factor for liver cirrhosis: a systematic review and meta-analysis. Drug Alcohol Rev 29: 437-45.

Rotily M, Durbec JP, Berthezene P, et al. (1990) Diet and alcohol in liver cirrhosis: a case-control study. Eur J Clin Nutr 44:595-603.

Safdar K, Schiff ER. (2004) Alcohol and hepatitis C. Semin Liver Dis 24: $305-15$.

Sandahl TD, Jepsen P, Thomsen KL, et al. (2011) Incidence and mortality of alcoholic hepatitis in Denmark 1999-2008: a nationwide population based cohort study. J Hepatol 54:760-4.

Saverymuttu SH, Joseph AE, Maxwell JD. (1986) Ultrasound scanning in the detection of hepatic fibrosis and steatosis. Br Med J (Clin Res Ed), 292, $13-5$.

Seitz HK, Egerer G, Simanowski UA, et al. (1993) Human gastric alcohol dehydrogenase activity: effect of age, sex, and alcoholism. Gut 34:1433-7.

Seitz HK, Stickel F. (2007) Molecular mechanisms of alcohol-mediated carcinogenesis. Nat Rev Cancer 7:599-612.

Sherman HI, Hardison JE. (1979) The importance of a coexistent hepatic rub and bruit. A clue to the diagnosis of cancer in the liver. JAMA 241:1495.

Sookoian S, Pirola CJ. (2016) How safe is moderate alcohol consumption in overweight and obese individuals? Gastroenterology 150:1698-703.e2.

Sorbi D, Boynton J, Lindor KD. (1999) The ratio of aspartate aminotransferase to alanine aminotransferase: potential value in differentiating nonalcoholic steatohepatitis from alcoholic liver disease. Am J Gastroenterol 94:1018-22.

Stewart SH. (2002) Racial and ethnic differences in alcohol-associated aspartate aminotransferase and gamma-glutamyltransferase elevation. Arch Intern Med 162:2236-9.

Stickel F, Hampe J. (2012) Genetic determinants of alcoholic liver disease. Gut 61:150-9.

Stinson FS, Grant BF, Dufour MC. (2001) The critical dimension of ethnicity in liver cirrhosis mortality statistics. Alcohol Clin Exp Res 25:1181-7.

Szabo G. (2017) Clinical trial design for alcoholic hepatitis. Semin Liver Dis $37: 332-42$.

Szabo G, Bala S. (2010) Alcoholic liver disease and the gut-liver axis. World J Gastroenterol 16:1321-9.

Szabo G, Petrasek J, Bala S. (2012) Innate immunity and alcoholic liver disease. Dig Dis 30:55-60.

Takahashi H, Ono M, Hyogo H, et al. (2015) Biphasic effect of alcohol intake on the development of fatty liver disease. J Gastroenterol 50:1114-23.

Teli MR, Day CP, Burt AD, et al. (1995) Determinants of progression to cirrhosis or fibrosis in pure alcoholic fatty liver. Lancet 346:987-90.

Thursz MR, Richardson P, Allison M, et al. (2015) Prednisolone or pentoxifylline for alcoholic hepatitis. N Engl J Med 372:1619-28.

Wechsler H, Austin SB. (1998) Binge drinking: the five/four measure. J Stud Alcohol 59:122-4.

Worner TM, Lieber CS. (1985) Perivenular fibrosis as precursor lesion of cirrhosis. JAMA 254:627-30.

Yamini D, Lee SH, Avanesyan A. (2014) Utilization of baclofen in maintenance of alcohol abstinence in patients with alcohol dependence and alcoholic hepatitis with or without cirrhosis. Alcohol Alcohol 49:453-6.

Yoon YH, Yi HY. (2006) Surveillance Report \#75: Liver Cirrhosis Mortality in the United States, 1970-2003. Bethesda, MD: National Institute on Alcohol Abuse and Alcoholism.

Zakhari S. (2013) Bermuda Triangle for the liver: alcohol, obesity, and viral hepatitis. J Gastroenterol Hepatol 28:18-25.

Zhong W, McClain CJ, Cave M, et al. (2010) The role of zinc deficiency in alcohol-induced intestinal barrier dysfunction. Am J Physiol Gastrointest Liver Physiol 298:G625-33. 